\title{
Influencia de una estrategia educativa de salud en el nivel de conocimientos y uso de métodos anticonceptivos, en estudiantes del tercer, cuarto y quinto año de secundaria de la Institución Educativa Enrique Paillardelle del Distrito Gregorio Albarracin Lanchipa. Junio - Diciembre, 2016
}

\section{Influence of an educational}

health strategy in the level of

knowledge and use of

contraceptive methods, in

students of the third, fourth and

fifth year of high school of the

Educational Institution Enrique

Paillardelle of the District

Gregorio Albarracin Lanchipa. June - December, 2016

\section{Edgard Guido Calderón Copa ${ }^{1}$ Juan José Evaristo Changllio Roas ${ }^{2}$ Yanela Elizabeth Paredes Espejo ${ }^{3}$ Kátherin Greiss Galván Jiménez ${ }^{4}$ Luis Cristian Inquilla Urbano 5 José Luis Llamoca Humpiri ${ }^{5}$}

1. Magíster en Ciencia y Tecnología de Alimentos. Especialista en Medicina Alternativa y Complementaria. Docente de la Facultad de Ciencias de la Salud. UNJBG

2. Doctor en Epidemiología. Docente de la Facultad de Ciencias de la Salud. UNJBG

3. Magíster en Salud Pública. Especialista en Farmacia Clínica y Atención Farmacéutica. Docente de la Facultad de Ciencias de la Salud. UNJBG

4. Ingeniera Biotecnóloga. Egresada de la Maestría de Medio Ambiente y Desarrollo Sostenible. Docente de CIMA

5. Estudiantes de la E.P. Farmacia y Bioquímica. UNJBG

\section{RESUMEN}

INTRODUCCIÓN: La Organización Mundial de la Salud considera a los adolescentes, un grupo de riesgo en materia sexual, porque no se protegen adecuadamente con anticonceptivos, lo que puede ocasionar embarazos no deseados y contagio de enfermedades ETS o el VIH. La información dada en instituciones educativas, no es suficiente para disminuir la prevalencia de conductas de riesgo para su salud. El objetivo fue determinar la influencia de la aplicación de una estrategia educativa de salud, en el nivel de conocimientos y uso de métodos anticonceptivos en estudiantes del tercer, cuarto y quinto año de secundaria de la Institución Educativa Enrique Paillardelle del Distrito Gregorio Albarracín Lanchipa. MATERIAL Y MÉTODOS: Investigación aplicada, experimental, prospectivo. La muestra

\section{ABSTRACT}

INTRODUCTION: The World Health Organization considers adolescents a risk group in sexual matters because they are not adequately protected with contraceptives, which can lead to unwanted pregnancies and the spread of STDs or HIV. The information given in educational institutions is not enough to reduce the prevalence of risk behaviors for their health. The objective was to determine the influence of the application of a health educational strategy on the level of knowledge and use of contraceptive methods in students of the third, fourth and fifth year of high school of the Enrique Paillardelle Educational Institution of the Gregorio Albarracín Lanchipa District. MATERIAL AND METHODS: Applied, experimental, prospective research. The sample consisted of 163 students of the 3 rd, 4 th and 5 th year 
estuvo conformada por 163 estudiantes del 3er, 4to y 5to año de secundaria de la Institución Educativa Enrique Paillardelle, la información se obtuvo de junio a diciembre del año 2016. RESULTADOS: El $92 \%$ de los estudiantes tuvo un nivel de conocimientos sobre planificación familiar adecuado después de la capacitación. CONCLUSIÓN: Existe influencia de la aplicación de una estrategia educativa de salud sobre el nivel de conocimientos en el uso de métodos anticonceptivos en los estudiantes de la Institución Educativa Enrique Paillardelle del Distrito Gregorio Albarracín Lanchipa de Tacna.

PALABRAS CLAVE: Métodos anticonceptivos, Estrategia educativa.

\section{INTRODUCCIÓN}

La población mundial de adolescentes ha ascendido a más de cien millones y en los países de desarrollo uno de cada cuatro personas está en la adolescencia a diferencia de uno de cada siete en los países desarrollados. El embarazo en edades cada vez más temprana se está convirtiendo en un problema social y de salud pública de alcance mundial. Afecta a todos los estratos sociales, pero predomina en la clase de bajo nivel socioeconómico, en parte debido a la falta de educación sexual, y al desconocimiento de los métodos de control de la natalidad (3).

El tema de la adolescencia siempre ha sido de mucho interés, solamente el hecho de que 1,2 mil millones de personas en el mundo estén entre las edades de 11 a 19 años de edad y de que la población joven en América latina y el Caribe ascienda a los 148 millones, así lo reclama. En el mundo la actividad sexual en este grupo de edades va creciendo, por lo que se considera, que la fecundidad en esta etapa constituye un problema de salud importante (4).

El objetivo fue determinar la influencia de la aplicación de una estrategia educativa de salud, en el nivel de conocimientos y uso de métodos anticonceptivos en estudiantes del tercer, cuarto y quinto año de secundaria de la Institución Educativa Enrique Paillardelle del Distrito Gregorio Albarracín Lanchipa; de junio a diciembre, 2016.

\section{MATERIAL Y MÉTODOS}

El Tipo de la Investigación es Aplicada, con Diseño: Experimental prospectivo y Nivel de la Investigación: Experimental. of secondary school of the Enrique Paillardelle Educational Institution, the information was obtained from June to December 2016. RESULTS: 92\% of the students had a level of knowledge about planning adequate family after the training. CONCLUSION: There is influence of the application of a health educational strategy on the level of knowledge in the use of contraceptive methods in the students of the Enrique Paillardelle Educational Institution of the Gregorio Albarracín Lanchipa District of Tacna.

KEYWORDS: Contraceptivemethods, Educationalstrategy.

Los principales métodos que se utilizaron fueron: Científico, Analítico, Sintético, Inductivo y Deductivo.

La Población estuvo conformada por los estudiantes del 3ro, 4to y 5 to año de secundaria de la Institución Educativa Enrique Paillardelle. Con respecto a la población de estudiantes se realizó un muestreo estratificado.

El tamaño de la muestra se calculó en base a la fórmula de Cochram para poblaciones siendo un total de 163 estudiantes encuestados y capacitados.

Para ello se utilizó como instrumento el cuestionario el que fue estructurado en base a las dimensiones e indicadores de las variables en estudio. Los resultados obtenidos se trabajaron estadísticamente utilizando tablas de frecuencias y gráficos de pastel.

\section{RESULTADOS}

\section{ANTES DE LA APLICACIÓN DE UNA ESTRATEGIA EDUCATIVA-CAPACITACIÓN}

\begin{tabular}{|c|c|c|}
\hline Conocimientos sobre Planificación Familiar & $\mathbf{N}^{\circ}$ & $\%$ \\
\hline Forma en que educas a la familia & 20 & 12,00 \\
\hline Reunirse en familia & 2 & 1,00 \\
\hline $\begin{array}{l}\text { Control de la reproducción mediante el uso de } \\
\text { métodos anticonceptivos }\end{array}$ & 130 & 80,00 \\
\hline Ayuda y unión familiar & 11 & 7,00 \\
\hline Total & 163 & 100,00 \\
\hline
\end{tabular}


En la tabla 1 observamos que el $80,00 \%$ de los estudiantes refieren que la planificación familiar es el control de la reproducción mediante el uso de métodos anticonceptivos; mientras, el $12,00 \%$ indican que la planificación familiar es la forma en la que educas a la familia.

\begin{tabular}{|c|c|c|}
\hline \multicolumn{3}{|c|}{$\begin{array}{l}\text { TABLA No } 2 \\
\text { Estudiantes según conocimientos sobre métodos } \\
\text { anticonceptivos. }\end{array}$} \\
\hline $\begin{array}{l}\text { Conocimientos sobre métodos } \\
\text { anticonceptivos }\end{array}$ & $\mathbf{N}^{\circ}$ & $\%$ \\
\hline Para no tener hijos & 71 & 44,00 \\
\hline Para cuidarse de enfermedades & 49 & 30,00 \\
\hline Permite preservar la salud & 36 & 22,00 \\
\hline No sabe & 7 & 4,00 \\
\hline Total & 163 & 100,00 \\
\hline
\end{tabular}

Respecto a los conocimientos sobre métodos anticonceptivos el $44,00 \%$ de estudiantes refieren que los métodos anticonceptivos se utilizan para no tener hijos; mientras, el $30,00 \%$ indican que se emplean para cuidarse de enfermedades.

\begin{tabular}{|c|c|c|}
\hline $\begin{array}{l}\text { Conocimiento sobre tipos de métodos } \\
\text { anticonceptivos }\end{array}$ & $\mathbf{N}^{\circ}$ & $\%$ \\
\hline Preservativo, píldora, inyectable & 63 & 39,00 \\
\hline Método del ritmo, píldora & 32 & 20,00 \\
\hline Condón, píldora & 15 & 9,00 \\
\hline Pastillas anticonceptivas, condón & 46 & 28,00 \\
\hline No sabe & 7 & 4,00 \\
\hline Total & 163 & 100,00 \\
\hline
\end{tabular}

De los estudiantes encuestados el $39,00 \%$ refieren que un método anticonceptivo es el preservativo, píldora, inyectable seguido del $28,00 \%$ que indican a las pastillas anticonceptivas y condón como otro de los métodos.

\section{DESPUÉS DE LA APLICACIÓN DE LA ESTRATEGIA EDUCATIVA-CAPACITACIÓN}

$\begin{array}{lcc}\text { TABLA No } 4 & \\ \text { Estudiantes según conocimientos sobre planificación } \\ \text { familiar. }\end{array}$

En la tabla 4, observamos que el 92,00 \% de los estudiantes refieren que la planificación familiar es el control de la reproducción mediante el uso de métodos anticonceptivos; mientras, el 8,00 \% indican que la planificación familiar es la forma en la que educas a la familia.

\begin{tabular}{|c|c|c|}
\hline \multicolumn{3}{|l|}{$\begin{array}{l}\text { TABLA No } 5 \\
\text { Estudiantes según conocimientos sobre métodos } \\
\text { anticonceptivos. }\end{array}$} \\
\hline Conocimientos sobre métodos anticonceptivos & $\mathbf{N}^{\circ}$ & $\%$ \\
\hline Dispositivo o medicación para impedir un embarazo & 119 & 73,00 \\
\hline $\begin{array}{l}\text { Hormonas, píldoras o dispositivo para evitar } \\
\text { enfermedades de trasmisión sexual }\end{array}$ & 42 & 26,00 \\
\hline No sabe & 2 & 1,00 \\
\hline Total & 163 & 100,00 \\
\hline
\end{tabular}

Respecto a los conocimientos sobre métodos anticonceptivos el $73,00 \%$ de estudiantes refieren que los métodos anticonceptivos son dispositivos 0 medicamentos para impedir un embarazo; mientras que, el $26,00 \%$ indican que son hormonas, píldoras o dispositivos para evitar enfermedades de trasmisión sexual.

\section{TABLA № 6}

Estudiantes según métodos anticonceptivos que conocen.

$\begin{array}{lrr}\begin{array}{l}\text { Conocimiento sobre tipos de métodos } \\ \text { anticonceptivos }\end{array} & \mathbf{N}^{\circ} & \% \\ \text { Preservativo, píldora, inyectable } & 67 & 41,00 \\ \text { Método del ritmo, píldora } & 32 & 20,00 \\ \text { Condón, píldora } & 15 & 9,00 \\ \text { Pastillas anticonceptivas, condón } & 49 & 30,00 \\ \text { No sabe } & 0 & 0,00 \\ \text { Total } & 163 & 100,00\end{array}$

De los estudiantes encuestados, el 41,00 \% refieren que los métodos anticonceptivos que conocen son el uso de los preservativos, píldora, inyectable; seguido del 30,00 \% que indican a las pastillas anticonceptivas y condón como otro de los métodos.

\section{DISCUSIÓN}

El presente trabajo de Investigación determina la influencia de la aplicación de una estrategia educativa de salud, en el nivel de conocimientos y uso de métodos anticonceptivos en estudiantes del tercer, cuarto y quinto año de secundaria de la Institución Educativa Enrique Paillardelle del Distrito Gregorio Albarracín Lanchipa, junio - diciembre; 2016, para evidenciar si los estudiantes tienen conocimiento sobre el uso de métodos anticonceptivos, 
seguidamente capacitarlos para mejorar dichos conocimientos y evitar desde ya la irresponsabilidad de ser padres tan jóvenes.

Debemos saber también que, en cuanto al ámbito sexual, se ha visto un incremento y un mayor adelanto en el inicio de la actividad sexual de los adolescentes, lo que ha provocado un aumento en la incidencia de embarazos en adolescentes (5).

La información que se proporciona en la institución educativa a los estudiantes, no es suficiente para disminuir la prevalencia de conductas de riesgo para su salud, ya que no aprovechan en forma efectiva las actividades o programas relacionados con la educación sexual y/o salud reproductiva, debido a que la información que se les brinda es igual al de la comunidad y no a la característica propia de su etapa evolutiva.

En la tabla 2, respecto a los conocimientos sobre métodos anticonceptivos el $44,00 \%$ de estudiantes refieren que los métodos anticonceptivos se utilizan para no tener hijos; mientras, el $30,00 \%$ indican que se emplean para cuidarse de enfermedades; por lo que observamos que la mayoría de los estudiantes no tiene un conocimiento adecuado sobre los métodos anticonceptivos y a ello apunta el presente trabajo de investigación.

En la tabla 3, el 39,00 \% refieren que el método anticonceptivo que conocen es el preservativo; píldora, inyectable seguido del $28,00 \%$ que indican a las pastillas anticonceptivas y condón como otro de los métodos. Por lo que observamos que la mayoría de los estudiantes conocen de algunos métodos anticonceptivos; sólo el 4,00\% indican que desconocen de algún método anticonceptivo.

A partir de la tabla 4 podemos observar los resultados después de haber realizado la capacitación sobre planificación familiar y los métodos anticonceptivos a los estudiantes. Es la tabla anteriormente mencionada se observa que el $92,00 \%$ de los estudiantes refieren que la planificación familiar es el control de la reproducción mediante el uso de métodos anticonceptivos; mientras que, el 8,00 \% indican que la planificación familiar es la forma en la que educas a la familia. Los estudiantes al recibir la información mediante la capacitación cambian sus conocimientos y por ello es necesario dicha capacitación y orientación sobre temas de sexualidad a los adolescentes.

En la tabla 5 respecto a los conocimientos sobre métodos anticonceptivos, el 73,00 \% de estudiantes refieren que los métodos anticonceptivos son dispositivos o medicamentos para impedir un embarazo; mientras que, el $26,00 \%$ indican que son hormonas, píldoras o dispositivos para evitar enfermedades de trasmisión sexual. De esta manera los conocimientos que adquieren los estudiantes pueden mejorar las prácticas y conductas en su vida sexual; siendo necesario realizar capacitaciones programadas tanto a los estudiantes, padres de familia y docentes.La educación juega un papel fundamental, ya que constituye la base del conocimiento y permite el desarrollo de las destrezas que habilitan, tanto al adolescente, como al joven para tomar decisiones y ser responsables en materia de salud. Por tanto, se hace necesaria una educación sexual de carácter alternativo que ofrezca a este grupo la posibilidad de elegir patrones y comportamientos en correspondencia con su forma particular de interpretar la sexualidad y determinar su manera de expresarla y sentirla de forma libre, lo que permite armonizar lo individual y lo social y enriquecerla sin conflictos antagónicos.La adolescencia y la juventud son edades aún tempranas, que resultan favorables para modificar enseñanzas rígidas, mitos, tabúes, creencias y formas de conductas que hayan influido en su formación, y así contribuir a que en su etapa de adultos manifiesten autorresponsabilidad en el cuidado de su salud. Los jóvenes en ocasiones carecen de los medios y la preparación para lograrlo, esto se comprueba en la poca percepción de riesgo, en la no protección en sus relaciones sexuales, en el cambio frecuente de parejas y en los embarazos no deseados (6).

En la tabla 6, observamos que de los estudiantes encuestados el $41,00 \%$ refieren que los métodos anticonceptivos que conocen son el uso de los preservativos, píldora, inyectable; seguido del 30,00 \% que indican a las pastillas anticonceptivas y condón como otro de los métodos. Por lo que al dar la capacitación la totalidad de los estudiantes respondieron que conocen algún método anticonceptivo.

Paredes (7) en Trujillo en el año 2010, en el estudio "Aplicación de talleres educativos para mejorar el nivel de conocimientos, en la prevención de ITS y VIH-SIDA en escolares de gran unidad escolar José Faustino Sánchez Carrión Trujillo - 2010", concluyó que con respecto al nivel de conocimientos antes de la aplicación de los talleres educativos, una mayor proporción de la población en estudio se encontraba en el nivel malo (50\%), siguiéndole en frecuencia el nivel regular con un $42,9 \%$, en tanto que después de la 
aplicación del taller educativo, existe seis veces más población en estudio en el nivel bueno $57,1 \%$, no encontrándose nadie en el nivel malo. Comparando el estudio realizado el $20 \%$ de los estudiantes tienen un nivel de conocimiento malo con respecto a conocimientos sobre planificación familiar; y después de la aplicación de la estrategia educativa el porcentaje disminuye a un $8 \%$.

Mantilla (8) en Lima en el año 2015, en el estudio “Nivel de Conocimiento sobre MétodosAnticonceptivos en las adolescentesentre 11 y 19 años del consultoriode Gineco-Obstericia del HNHU,octubre -noviembre", se concluyó que el $40 \%$ de los adolescentes dijo utilizar preservativo o condón, el $28,67 \%$ pastillas anticonceptivas, el $10,67 \%$ método del ritmo y solo el $20,67 \%$ no utiliza métodos anticonceptivos. El $82 \%$ obtuvo un nivelde conocimiento sobre métodos anticonceptivos excelente, $11,33 \%$ bueno, 6,67\% regulary $0 \%$ deficiente. Con respecto al trabajo realizado podemos mencionar que el $92 \%$ de los estudiantes tuvo un nivel de conocimientos sobre planificación familiar adecuado después de la capacitación.

Posada (9) en Costa Rica en el año 2015, en el trabajo de investigación "Conocimientos previos acerca de métodos anticonceptivos y su relación con conocimientos adquiridosdespués de una intervención educativa con simulador". Se detectó que los presaberes en métodos anticonceptivos son deficientes independientemente de laedad y la escolaridad, lo cual requiere planeación, desarrollo y evaluación de la estrategia de aprendizaje colectivoy cooperativo mediante talleres. En cuanto a los post-saberes, se obtuvo una calificación de deficientes aregulares, en el caso del condón masculino, mientras que el reconocimiento de métodos modernos como parche,anillo vaginal, dispositivo intrauterino, entre otros, se dio gracias al contacto directo con el simulador.

Concluimos que a pesar de las diferencias de edad y escolaridad, no hubo hallazgos relevantes quedemostraran diferencias de conocimiento. En los pre-saberes, se encontró niveles de conocimiento deficientessobre métodos anticonceptivos. El uso de simuladores como herramienta de aprendizaje demostró eficacia en lostalleres; en cuanto a los simuladores posibilitan la ruptura de la barrera cognitiva, dado que el adolescente, en estecaso, puede explorar y adquirir conocimiento al interaccionar con el objeto de aprendizaje. Sin embargo, en el trabajo realizado el nivel de conocimientos y uso de anticonceptivos en estudiantes de la Institución Educativa Enrique Paillardelle, antes de la aplicación de la estrategia educativa era inadecuado y después de la capacitación se evidenció un conocimiento adecuado.

Finalmente existe influencia de la aplicación de una estrategia educativa de salud sobre el nivel de conocimientos, y el uso de métodos anticonceptivos en los estudiantes del tercer, cuarto y quinto año de secundaria de la Institución Educativa Enrique Paillardelle del Distrito Gregorio Albarracín Lanchipa, conclusión acorde con la hipótesis planteada. De igual manera, la mayoría de los estudiantes de la Institución Educativa son varones (52,0\%), y las edades que más predominan en los estudiantes se encuentran entre los 15 y 17 años (31,0\%), edades acordes a los grados que les corresponde a los estudiantes. Asimismo, el nivel de conocimientos y uso de anticonceptivos en estudiantes del tercer, cuarto y quinto año de secundaria de la Institución Educativa, antes de la aplicación de la estrategia educativa en salud es inadecuado. Finalmente, el nivel de conocimientos y uso de anticonceptivos en estudiantes de la Institución Educativa después de la aplicación de la estrategia educativa en salud es adecuado.

Recomendamos que se deben realizar campañas sobre el uso correcto y funcionamiento de los métodos anticonceptivos, así como de las enfermedades de transmisión sexual en los planteles educativos, con el fin de que los jóvenes cuenten con información profesional, y así colaborar a reducir las altas tasas de abortos, mortalidad materna infantil y embarazos no deseados. De igual manera utilizar los medios de comunicación,como medios de información más usados para dar a conocer los distintos métodos anticonceptivos de esta manera transmitir información científicamente validada a los adolescentes. Finalmente organizar a todas las Escuelas Profesionales de la Facultad de Ciencia de la Salud de la Universidad Nacional Jorge Basadre Grohmann de Tacna durante el año académico escolar con la finalidad de reforzar los conocimiento sobre el uso de los distintos métodos anticonceptivos mediante capacitaciones constantes en todos los colegios de Tacna, especialmente en estudiantes de tercer, cuarto y quinto año de secundaria, con la finalidad de reducir las altas tasas de abortos, mortalidad materno infantil y embarazos no deseados. 


\section{REFERENCIAS BIBLIOGRÁFICAS}

1. Peláez J. Anticoncepción en los adolescentes y adultos jóvenes. La Habana: Científico - Técnica; 2001.

2. Castro P. Mercedes PMA y PWJ. Conocimientos, mitos y prácticas sobre el uso de métodos anticonceptivos asociados al incremento de embarazos no planificados en estudiantes adolescentes en dos Instituciones Educativas en la ciudad de Cartagena. 2012. Tesis maestría. Cartagena: Corporación Universitaria Rafael Nuñez, Departamento de Posgrado; 2012.

3. Pérez L, Gil O, \& Hernández, M. Comportamiento del embarazo en la adolescencia en un grupo de estudiantes de la enseñanza media. 2005.

4. Lugones M, Ramírez M, Machado H. Intervención educativa en adolescentes atendidas en consulta de ginecología infanto juvenil. Cubano Pediátrica. 2008.

5. Castro P, Mercedes PM. Conocimientos, mitos y prácticas sobre el uso de métodos anticonceptivos asociados al incremento de embarazos no planificados en estudiantes adolescentes en dos Instituciones Educativas en la ciudad de
Cartagena. 2012. Tesis maestría, Corporación Universitaria Rafael Nuñez, Departamento de Posgrado, Cartagena. 2012.

6. Torres M. Novios violentos en un grupo de adolescentes. Tesis, CENESEX, Habana. 2005.

7. Paredes W. Aplicación de talleres educativos para mejorar el nivel desconocimientos, en la prevención de ITS y VIH-SIDA en escolares de gran unidad escolar José Faustino Sánchez Carrión. Tesis profesional, Universidad Nacional de Trujillo, Departamento de Obstetricia, Trujillo. 2010.

8. Mantilla C. Nivel de Conocimiento sobre Métodos Anticonceptivos en las adolescentes entre 11 y 19 años del consultorio de Gineco-Obstericia del HNHU, Octubre Noviembre. Lima. 2015.

9. Posada M. Conocimientos previos acerca de métodos anticonceptivos y su relación con conocimientos adquiridosdespués de una intervención educativa con simulador. Costa Rica. 2015.

\section{Correspondencia \\ Edgard Guido Calderón Copa \\ edgardguidocald@gmail.com}

Fecha de recepción: 20 de setiembre de 2017 Fecha de aceptación: 20 de octubre de 2017 